\title{
Fatores Associados à Ocorrência de Hipertensão Arterial em Trabalhadores da Indústria do Estado do Rio Grande do Sul, Brasil
}

\author{
Factors Associated with the Occurrence of Arterial Hypertension in Industry Workers of State of Rio Grande do \\ Sul, Brazil

\begin{abstract}
Paula Brustolin Xavier, ${ }^{1,2}$ Anderson Garcez, ${ }^{1,3}$ (1) Gabriela Herrmann Cibeira, ${ }^{4}$ Antonino Germano, ${ }^{4}$ Maria Teresa Anselmo Olinto ${ }^{1,5}$

Programa de Pós-Graduação em Saúde Coletiva - Universidade do Vale do Rio dos Sinos (UNISINOS), ${ }^{1}$ São Leopoldo, RS - Brasil

Área de Ciências da Vida - Universidade do Oeste de Santa Catarina (UNOESC), ${ }^{2}$ Joaçaba, SC - Brasil

Programa de Pós-graduação em Ciências da Nutrição - Universidade Federal de Ciências da Saúde de Porto Alegre (UFCSPA), ${ }^{3}$ Porto Alegre, RS - Brasil Serviço Social da Indústria do Estado do Rio Grande do Sul (SESI-RS), ${ }^{4}$ Porto Alegre, RS - Brasil

Programa de Pós-graduação em Alimentação, Nutrição e Saúde - Universidade Federal do Rio Grande do Sul (UFRGS), ${ }^{5}$ Porto Alegre, RS - Brasil
\end{abstract}

\section{Resumo}

Fundamento: A hipertensão é um importante e persistente problema de saúde pública, sendo uma das principais causas de doenças cardiovasculares e mortalidade geral.

Objetivos: Este estudo buscou verificar a prevalência e os fatores associados à hipertensão arterial sistêmica em trabalhadores da indústria do estado do Rio Grande do Sul, Brasil.

Métodos: Trata-se de um estudo transversal com dados secundários de 20.792 industriários de 18 a 59 anos de idade. A presença de hipertensão arterial foi determinada a partir da pressão arterial sistólica $\geq 140 \mathrm{mmHg}$ e/ou pressão arterial diastólica $\geq 90 \mathrm{mmHg}$, ou estar fazendo uso de medicação anti-hipertensiva. Os fatores investigados incluíram características demográficas, socioeconômicas, comportamentais, de estado nutricional e de história familiar. Regressão de Poisson foi utilizada na análise multivariável, adotando-se um p<0,05 como nível de significância. Todas as análises foram estratificadas por sexo.

Resultados: A amostra incluiu 12.349 homens e 8.443 mulheres com média de idade geral de 32,8 anos (Desviopadrão=9,8 anos). A prevalência de hipertensão foi de 10,3\% (IC95\%:9,8-10,7), sendo esta significativamente maior entre os homens do que entre as mulheres $(10,9 \%$ vs $9,4 \% ; p=0,001)$. A hipertensão mostrou-se associada à elevação da faixa etária, baixa escolaridade, viver com companheiro, ter sobrepeso ou obesidade, e ter pelo menos um parente com história de hipertensão para ambos os sexos. As mulheres com melhores condições socioeconômicas apresentaram menores prevalências de hipertensão.

Conclusões: Os principais fatores associados à hipertensão arterial compreenderam características sociodemográficas, nutricionais e de história familiar. Ademais, as condições socioeconômicas demonstraram uma associação com a ocorrência de hipertensão, principalmente entre as mulheres.

Palavras-chave: Hipertensão; Fatores de Risco; Epidemiologia; Hereditariedade; Obesidade; Trabalhadores; Indústrias.

\footnotetext{
Abstract

Background: Hypertension is a serious and persistent public health problem and is one of the main causes of cardiovascular diseases and general mortality.

Objectives: This study aimed to verify the prevalence and factors associated with systemic arterial hypertension in workers from the state of Rio Grande do Sul, Brazil.

Methods: This is a cross-sectional study using the secondary data from 20,792 industry workers from 18 to 59 years of age. The presence of arterial hypertension was determined from systolic blood pressure $\geq 140 \mathrm{mmHg}$ and/or diastolic blood pressure $\geq 90 \mathrm{mmHg}$ or taking

Correspondência: Maria Teresa Anselmo Olinto •

Programa de Pós-Graduação em Saúde Coletiva - Universidade do Vale do Rio dos Sinos (UNISINOS) Av. Unisinos 950, C. P. 275.

CEP 93022-000, São Leopoldo, RS - Brasil

E-mail: mtolinto@gmail.com

Artigo recebido em 18/11/2019, revisado em 07/08/2020, aceito em 09/09/2020.
}

DOI: https://doi.org/10.36660/abc.20190815 
antihypertensive medication. Factors investigated included demographic, socioeconomic, behavioral, nutritional status, and family history characteristics. Poisson regression was used in multivariate analysis, adopting a significance level of $p<0.05$. All analyses were stratified by sex.

Results: The sample included 12,349 men and 8,443 women with a mean age of 32.8 years (Standard Deviation = 9.8). The prevalence of arterial hypertension was 10.3\% (95\% Cl: 9.8-10.7), which was significantly higher in men than in women (10.9\% vs 9.4\%; p = 0.001). Arterial hypertension was associated with increased age, a low level of education, living with a partner, being overweight or obese, and having at least one relative with a history of hypertension for both sexes. Women with better socioeconomic conditions presented a lower prevalence of hypertension.

Conclusions: The main factors associated with hypertension included sociodemographic, nutritional, and family history characteristics. In addition, socioeconomic conditions showed an association with the occurrence of hypertension, especially among women.

Keywords: Hypertension; Risk Factors; Epidemiology; Heredity; Obesity; Workers; Industry.

Full texts in English - http://www.arquivosonline.com.br

\section{Introdução}

A Hipertensão Arterial Sistêmica (HAS) é um importante problema de saúde pública, além de ser um fator de risco para doenças cardiovasculares. ${ }^{1-4}$ Estima-se que a prevalência de HAS na população mundial seja em torno de $22 \%{ }^{5}$ Na América Latina, o Brasil apresenta uma das maiores prevalências de HAS, com diferença significativa entre homens $(26,7 \%)$ e mulheres (19,9\%). ${ }^{6}$ Em trabalhadores da indústria, a prevalência de HAS diverge conforme a localização geográfica, sendo mais prevalente na região Nordeste $(35,1 \%)$, seguido do Centro-Oeste (19\%) e Sul do Brasil (19,8\%).7

Potenciais fatores de risco para a ocorrência de HAS têm sido explorados em diferentes grupos populacionais de trabalhadores, evidenciando-se uma forte influência de fatores sociodemográficos, comportamentais e ocupacionais. ${ }^{7-9}$ A HAS tem sido associada principalmente aos hábitos comportamentais como o consumo de álcool e tabaco, dieta desequilibrada, inatividade física, além do possível efeito do histórico familiar da doença. ${ }^{10}$ Dessa forma, hábitos de vida não saudáveis também podem influenciar na ocorrência de outras doenças associadas a HAS, especialmente quando associados ao histórico familiar e presença de obesidade. ${ }^{9}$

Os fatores de risco cardiometabólicos normalmente são assintomáticos, e identificá-los precocemente permite que medidas e ações de prevenção possam ser estabelecidas. ${ }^{11}$ Além disso, os diferentes cenários ocupacionais e formas de gestão produtiva têm incorporado mudanças no cotidiano dos trabalhadores. ${ }^{12}$ Apesar de estudos prévios terem investigado a ocorrência de hipertensão entre trabalhadores da indústria no Brasil, ${ }^{8,13}$ não se identificaram estudos conduzidos no estado do Rio Grande do Sul, um dos estados mais industrializados do país. Dessa forma, o presente estudo buscou verificar a prevalência e investigar os fatores associados com a HAS em trabalhadores do setor da indústria do estado do Rio Grande do Sul, Brasil.

\section{Métodos}

Estudo transversal com dados secundários do "Projeto Coração" do Serviço Social da Indústria do Rio Grande do Sul (SESI-RS), realizado no período de 2006 a 2009. A amostra contemplou trabalhadores adultos com 18 a 59 anos de idade e que atuavam em indústrias de pequeno (20 - 99 empregados), médio (100 - 499 empregados) e grande porte
(> 500 empregados), distribuídas pelas regiões de maior industrialização do estado do Rio Grande do Sul. Embora o presente estudo tenha utilizado dados secundários, este foi submetido (CAAE: 90968018.9.0000.5344/2018) e aprovado pelo Comitê de Ética em Pesquisa da Universidade do Vale do Rio dos Sinos (Parecer n. 2.719.764). Dessa forma, a preservação do anonimato dos trabalhadores foi estabelecida junto ao termo de responsabilidade submetido à instituiçãofonte dos dados.

\section{Amostragem}

No sentido de garantir uma representatividade dos trabalhadores da indústria do Estado, e considerando a impossibilidade de relacionar todos os empregados, um processo de amostragem em duas etapas foi realizado. $\mathrm{Na}$ primeira, foi selecionada uma amostra aleatória simples das indústrias. Na segunda, obteve-se uma amostra de empregados registrados nas indústrias selecionadas na primeira etapa. A seleção das empresas foi realizada por meio de levantamento da sua localização (municípios) e número total de empregados, incluindo todas as atividades econômicas da Relação Anual de Informações Sociais (RAIS), ano-base 2004. Para determinar quais municípios deveriam ser incluídos na pesquisa, foi organizada uma lista contemplando os municípios que concentravam $80 \%$ dos empregos industriais do estado, distribuídos segundo o tamanho da empresa e Classificação Nacional de Atividades Econômicas (CNAEs). Por meio de escolha aleatória simples e estratificada segundo porte, foram sorteadas 145 empresas, contemplando indústrias do setor de alimentos e bebidas (CNAE 10 e 11), de produtos de couro (CNAE 15), de produtos metalmecânicos (CNAEs 24, 25, 28 e 29), e de fumo (CNAE 12). Esse processo visou contemplar uma amostra representativa em relação à população de empresas, considerando um nível de confiança de 95\%, uma variância para o estimador na proporção de 0,25, e um erro máximo nas estimativas de proporções de 3,6 pontos percentuais. Posteriormente, obteve-se uma amostra proporcional ao número total de trabalhadores registrados nas empresas. Assim, ao final das duas etapas, contemplou-se a avaliação de 21.341 trabalhadores.

\section{Coleta de dados e instrumentos}

A coleta de dados ocorreu por meio de entrevistas presenciais, durante o horário de trabalho e incluindo 
todos os turnos de trabalho existentes na empresa. Para garantir a padronização, todos os entrevistadores receberam treinamento sobre os procedimentos adequados para a coleta dos dados. Todas as entrevistas e avaliações foram realizadas dentro das dependências das empresas, sendo que os trabalhadores foram previamente liberados pelas empresas. Além disso, a adesão dos trabalhadores foi totalmente voluntária e consentida.

Foi utilizado um questionário padronizado, pré-codificado e pré-testado. O questionário contemplou informações demográficas, socioeconômicas, comportamentais, de estado nutricional, e de histórico familiar. As características demográficas investigadas foram: sexo, idade (faixas etárias de 10 anos), cor da pele (brancas e não-brancas) e situação conjugal (com e sem companheiro). Dentre as socioeconômicas, incluíram-se renda familiar mensal (estratificada em valores absolutos de reais) e escolaridade (estratificada em grau de ensino).

Quanto às características comportamentais, investigouse tabagismo (fumante, não fumante e ex-fumante), considerando-se "fumante" o trabalhador que estivesse em uso de qualquer tipo ou quantidade de tabaco, diariamente, por ao menos seis meses. "Ex-fumante" foi definido como aquele que, tendo sido fumante, não tenha feito uso de tabaco nos últimos seis meses; e "não fumante" aquele que nunca fez uso de tabaco em qualquer período da vida. Já o consumo de álcool foi definido com base no consumo regular de qualquer tipo de bebida alcoólica em, no mínimo, uma vez por semana. Outra característica comportamental investigada foi a prática de atividade física regular, em que os participantes foram divididos entre ativos e inativos, considerando-se como atividade física regular aquelas praticadas de forma regular e constante.

O estado nutricional foi avaliado por meio do Índice de Massa Corporal - IMC, obtido pelas medidas de peso e altura, considerando a seguinte equação: peso (em quilogramas) dividido pela altura (em metros) ao quadrado. Foram classificados com sobrepeso todos os trabalhadores com IMC entre 25,0 e $29,9 \mathrm{~kg} / \mathrm{m}^{2}$, e com obesidade todos aqueles com $30,0 \mathrm{~kg} / \mathrm{m}^{2}$ ou mais. ${ }^{14}$ Para a mensuração do peso corporal em quilogramas, foi utilizada balança digital previamente calibrada. A aferição da altura em centímetros foi realizada por meio de um estadiômetro móvel mantido sobre piso plano, sem rodapé e apoiado na parede. As medidas foram realizadas com os trabalhadores descalços, com roupas leves (sem casacos ou EPIs), totalmente eretos e com calcanhares unidos. O histórico familiar de hipertensão (mãe, pai e avós), foi relatado pelos participantes.

A variável "desfecho" foi obtida por meio da mensuração das pressões sistólicas e diastólicas, utilizando-se de estetoscópio e esfigmomanômetro do tipo aneroide, testados e calibrados pelo INMETRO (Instituto Nacional de Metrologia, Qualidade e Tecnologia). As aferições foram realizadas em duplicata, utilizando-se a média entre as duas medidas observadas. Todas as medidas foram obtidas no braço direito, com intervalo de 3 minutos, estando o indivíduo na posição sentada. Todas as mensurações foram executadas por estagiários de graduação em enfermagem. Estes foram previamente treinados, considerando a aplicação do protocolo de recomendações estabelecido pelo III Consenso Brasileiro de Hipertensão Arterial. ${ }^{15}$ As aferições foram realizadas, durante o horário de trabalho e previamente à aplicação dos questionários, em um espaço físico adequado e disponibilizado pelas empresas (afastado da área de produção e com baixos níveis de ruído).

A hipertensão arterial foi definida pela presença de pressão arterial sistólica $\geq 140 \mathrm{mmHg}$ e/ou pressão arterial diastólica $\geq 90 \mathrm{mmHg}$, ou estar fazendo uso de medicação anti-hipertensiva regular ou esporádica. ${ }^{4,16}$ Trabalhadores com diagnóstico prévio de HAS e sem tratamento atual (uso de medicação) foram classificados com base no nível de pressão arterial mensurado no presente estudo.

\section{Análise estatística}

Estatística descritiva foi utilizada para a distribuição geral da amostra e distribuição do desfecho em estudo, utilizando-se de frequências absoluta e relativa para as variáveis categóricas e média, com os seus respectivos desvios-padrões, para as variáveis numéricas. Para as análises brutas, entre a variável desfecho (HAS) e as variáveis de exposição (independentes), utilizou-se do teste do Qui-quadrado de Pearson para heterogeneidade de proporções (variáveis categóricas) ou de tendência linear (variáveis ordinais). Uma possível presença de colinearidade entre as variáveis de exposição foi avaliada por meio da associação entre elas.

Análise multivariável foi realizada por meio da Regressão de Poisson com variância robusta, ${ }^{17}$ considerando os valores de significância estatística obtidos por meio do teste Wald para heterogeneidade de proporções (variáveis categóricas) ou de tendência linear (variáveis ordinais). A análise multivariável baseou-se num modelo conceitual de determinação e das inter-relações das variáveis, ${ }^{18}$ considerando dois níveis hierárquicos de ajuste. No primeiro nível, foi realizada análise ajustada entre as características demográficas e socioeconômicas entre si. Já no segundo nível, foi realizada a análise ajustada incluindo as variáveis do primeiro nível com $p<0,20$, e as variáveis comportamentais, de estado nutricional e de história familiar. Todas as análises foram estratificadas por sexo, considerando a heterogeneidade da prevalência do desfecho (HAS). Todas as análises foram realizadas por meio do programa Stata versão 12 (StataCorp LP, College Station, Texas, USA), considerando-se um nível de significância de $5 \%(p<0,05)$.

\section{Resultados}

Dos 21.341 trabalhadores qualificados neste estudo, 489 $(2,3 \%)$ foram classificados como perdas ou exclusões por falta de informações ou por estarem fora da faixa etária alvo, e em 60 trabalhadores $(0,28 \%)$ não foi possível obter a medida de pressão arterial. Assim, um total de 20.792 trabalhadores com média de idade de 32,8 \pm 9,8 anos foi incluída na análise final, considerando 12.349 homens (59,4\%) e $8.443(40,6 \%)$ mulheres, com médias de idade de 33,5 \pm 10,1 anos e 31,9 \pm 9,3 anos, respectivamente. Em relação aos setores da indústria, o presente estudo incluiu 4.356 trabalhadores do setor de alimentos e bebidas (20,9\%), 9,692 do setor de produtos de couro (46,6\%), 6,168 do setor de produtos metalmecânicos $(29,7 \%)$, e 576 do setor de fumo $(2,8 \%)$. 


\section{Artigo Original}

Tabela 1 - Distribuição da amostra e prevalências de hipertensão arterial sistêmica (HAS $\geq 140 / 90$ mmHg ou tratamento) segundo características demográficas, socioeconômicas, comportamentais, estado nutricional e hereditárias, na amostra de homens e mulheres trabalhadores da indústria do estado do Rio Grande do Sul, RS, Brasil, 2006-2009. (N=20.792)

\begin{tabular}{|c|c|c|c|c|c|c|}
\hline \multirow[b]{2}{*}{ Características } & \multicolumn{3}{|c|}{ Homens (12.349) } & \multicolumn{3}{|c|}{ Mulheres (8.443) } \\
\hline & n (\%) & $\%$ HAS & p-valor & n (\%) & $\%$ HAS & p-valor \\
\hline Idade (anos) & & & $<0,001$ & & & $<0,001$ \\
\hline 18 a 29 & $5.180(42,0)$ & 2,6 & & $3.925(46,5)$ & 2,0 & \\
\hline 30 a 39 & $3.613(29,2)$ & 9,5 & & $2.622(31,1)$ & 8,6 & \\
\hline 40 a 49 & $2.593(21,0)$ & 20,1 & & $1.522(18,0)$ & 22,6 & \\
\hline 50 a 59 & $963(7,8)$ & 35,5 & & $374(4,4)$ & 39,0 & \\
\hline Cor da pele & & & 0,936 & & & 0,508 \\
\hline Branca & $9.595(77,7)$ & 10,8 & & $7.031(83,3)$ & 9,5 & \\
\hline Não Branca & $2.754(22,3)$ & 10,9 & & $1.412(16,7)$ & 8,9 & \\
\hline Situação conjugal & & & $<0,001$ & & & $<0,001$ \\
\hline Sem companheiro & $3.976(32,2)$ & 5,8 & & $2.724(32,3)$ & 7,0 & \\
\hline Com companheiro & $8.373(67,8)$ & 13,2 & & $5.719(67,7)$ & 10,5 & \\
\hline Escolaridade & & & $<0,001$ & & & $<0,001$ \\
\hline Fundamental incompleto & $4.039(32,7)$ & 15,8 & & $3.469(41,1)$ & 14,3 & \\
\hline Fundamental completo & $2.932(23,8)$ & 8,6 & & $2.060(24,4)$ & 7,5 & \\
\hline Médio completo & $3.699(29,9)$ & 7,9 & & $2.021(24,0)$ & 5,4 & \\
\hline Superior incompleto/completo & $1.679(13,6)$ & 9,5 & & $893(10,6)$ & 3,6 & \\
\hline Renda familiar mensal (reais) & & & 0,004 & & & 0,002 \\
\hline$\leq 800$ & $2.973(25,3)$ & 10,6 & & $2.410(29,7)$ & 10,6 & \\
\hline 801 a 1.200 & $3.113(26,4)$ & 10,0 & & $2.819(34,7)$ & 9,5 & \\
\hline 1.201 a 1.800 & $2.518(21,3)$ & 10,0 & & $1.578(19,5)$ & 9,9 & \\
\hline$>1.800$ & $3.183(27,0)$ & 12,9 & & $1.313(16,2)$ & 7,1 & \\
\hline Tabagismo & & & $<0,001$ & & & 0,014 \\
\hline Não fumante & $9.051(73,3)$ & 9,8 & & $7.153(84,8)$ & 9,2 & \\
\hline Ex-fumante & $1.267(10,3)$ & 16,7 & & $519(6,1)$ & 12,9 & \\
\hline Fumante & $2.031(16,4)$ & 12,0 & & $771(9,1)$ & 8,4 & \\
\hline Consumo de álcool & & & 0,435 & & & 0,001 \\
\hline Não consome & $7.650(61,9)$ & 10,7 & & $7.483(88,6)$ & 9,8 & \\
\hline Consome & $4.699(38,1)$ & 11,1 & & $960(11,4)$ & 6,6 & \\
\hline Atividade física & & & $<0,001$ & & & 0,324 \\
\hline Ativo & $4.419(36,0)$ & 8,9 & & $2.054(24,5)$ & 9,9 & \\
\hline Inativo & $7.860(64,0)$ & 11,9 & & $6.347(75,5)$ & 9,2 & \\
\hline Estado nutricional (IMC) & & & $<0,001$ & & & $<0,001$ \\
\hline Normal $\left(<25 \mathrm{~kg} / \mathrm{m}^{2}\right.$ & $5.664(46,0)$ & 4,7 & & $4.538(53,9)$ & 3,6 & \\
\hline Sobrepeso (25 a $\left.29,9 \mathrm{~kg} / \mathrm{m}^{2}\right)$ & $5.014(40,6)$ & 13,0 & & $2.505(29,8)$ & 11,9 & \\
\hline Obeso $\left(\geq 30 \mathrm{~kg} / \mathrm{m}^{2}\right)$ & $1.660(13,5)$ & 25,4 & & $1.380(16,3)$ & 24,0 & \\
\hline $\begin{array}{l}\text { Presença de hipertensão } \\
\text { na família }\end{array}$ & & & $<0,001$ & & & $<0,001$ \\
\hline Não & $6.442(52,2)$ & 7,8 & & $3.477(41,2)$ & 4,8 & \\
\hline Mãe ou Pai & $4.987(40,5)$ & 12,5 & & $3.950(46,8)$ & 10,3 & \\
\hline Mãe e Pai & $674(5,5)$ & 22,9 & & $657(7,8)$ & 22,2 & \\
\hline Mãe, Pai e Avós & $226(1,8)$ & 25,7 & & $349(4,1)$ & 21,2 & \\
\hline
\end{tabular}

IMC: índice de massa corporal. p-valor para teste do Qui-quadrado para heterogeneidade de proporções (variáveis categóricas) ou tendência linear (variáveis ordinais) 
A Tabela 1 apresenta as características gerais da amostra para homens e mulheres e as respectivas prevalências de HAS conforme as variáveis investigadas. Na amostra geral, verificouse uma prevalência de hipertensão arterial (HAS $\geq 140 / 90$ mmHg ou tratamento) de 10,3\% (IC95\%: 9,8 - 10,7), sendo que $43,7 \%$ desses participantes não faziam uso de medicação anti-hipertensiva $(n=931)$. A prevalência de HAS foi maior nos homens (10,9\%; IC95\%:10,3 - 11,4) do que entre as mulheres (9,4\%; IC95\%: 8,8 - 10,0; $p=0,001)$, sendo que $24,3 \%$ dos homens e $25,8 \%$ das mulheres com idade acima de 40 anos apresentavam HAS. Verificou-se, também, que as mulheres com melhores condições socioeconômicas apresentaram menores prevalências de HAS quando comparado aos homens (Tabela 1). Não foi identificada colinearidade entre nenhuma das variáveis independentes, incluindo idade e estado marital, assim como entre IMC, renda familiar e escolaridade.

A Tabela 2 apresenta as razões de prevalências brutas e ajustadas, estratificadas entre homens e mulheres, para a ocorrência de HAS conforme os fatores investigados. Após análise ajustada, a prevalência de HAS mostrou-se associada à elevação da faixa etária, baixa escolaridade, viver com companheiro, ter sobrepeso ou obesidade, e ter pelo menos um familiar com história de hipertensão. Quanto aos fatores socioeconômicos, a menor escolaridade manteve-se associada com maiores prevalências de HAS apenas entre as mulheres. Associação diretamente proporcional foi observada entre estado nutricional e HAS em ambos os sexos. Ainda, a prevalência de HAS foi direta e significativamente maior entre aqueles que tinham um dos pais, ou ambos os pais e mais os avós, com histórico de hipertensão. Embora renda familiar, tabagismo, consumo de álcool e prática de atividade física tenham apresentado uma tendência de associação na análise bruta, estas foram contraditórias e todas perderam significância após o ajuste (Tabela 2).

\section{Discussão}

Nossos achados apontaram que fatores sociodemográficos, nutricionais e de história familiar estão associados com a ocorrência de HAS nos trabalhadores da indústria. Ademais, identificou-se que a prevalência de HAS aumentou conforme o avanço da idade e situação conjugal, em ambos os sexos. Além disso, as condições socioeconômicas desfavoráveis se associaram com a presença de HAS, principalmente entre as mulheres.

A prevalência de HAS verificada neste estudo ficou abaixo do verificado em estudos conduzidos com trabalhadores da indústria no Brasil. ${ }^{8,13}$ Essa variabilidade pode ser potencialmente explicada pelo período de coleta de dados do presente estudo (2006 a 2009), pela diversidade de critérios utilizados para definir a presença de HAS, além do tipo de aparelho e momento de aferição da pressão arterial sistêmica, dificultando as comparações entre os estudos. ${ }^{19}$ Ademais, os trabalhadores homens apresentaram uma maior prevalência de HAS, quando comparados às mulheres, convergindo com um estudo prévio conduzido com trabalhadores da indústria. ${ }^{7}$

Embora estudos transversais não permitam estabelecer relações de causa-efeito, é possível considerar que as alterações fisiológicas relacionadas ao avanço da idade podem elevar os riscos no sistema cardiovascular. , $20,21^{21}$ Neste estudo, a prevalência de HAS foi significativamente maior entre os trabalhadores com 40 anos ou mais de idade. Achado semelhante foi observado em um estudo conduzido com trabalhadores do setor de metalúrgica e siderúrgica, ${ }^{13}$ evidenciando que o trabalhador acima de quarenta anos é uma prioridade para ações de prevenção e intervenção para HAS.

Trabalhadores que relataram viver com companheiro(a) apresentaram uma maior prevalência de HAS. Achado semelhante foi previamente observado, em que viver com companheiro(a) esteve associado a presença de outras morbidades e HAS. ${ }^{22}$ Já ao analisar a influência da escolaridade na HAS, nosso estudo identificou que as mulheres com menor escolaridade apresentaram uma maior probabilidade para HAS, assemelhando-se a achados prévios em amostras de trabalhadores. ${ }^{23,24}$ Embora tenha-se buscado identificar diferenças entre os potencias fatores de risco para HAS entre trabalhadores de ambos os sexos, destaca-se que a suscetibilidade biológica relacionada ao sexo ou a diferenças de gênero, seja desvinculada dos riscos de saúde inerentes ao processo de trabalho para ambos os sexos. ${ }^{25}$ Contudo, cabe destacar que as mulheres muitas vezes ocupam os cargos de menor prestígio e com menores salários nas empresas, ${ }^{26}$ aspectos que podem refletir nas maiores prevalências de HAS em mulheres de menor posição socioeconômica.

Neste estudo, os trabalhadores com sobrepeso ou obesidade apresentaram uma probabilidade de duas a três vezes maior de ter HAS. Estudos prévios corroboram uma associação significativa entre estado nutricional e HAS, incluindo estudos conduzidos com trabalhadores. ${ }^{12,20,27,28}$ Esse achado demonstra uma possível relação entre o aumento da prevalência da HAS com a transição nutricional da população. ${ }^{6,28}$ Já no que se refere ao histórico familiar de HAS, verificamos uma prevalência de HAS significativamente maior entre aqueles trabalhadores que reportaram história familiar pregressa de HAS em pai, mãe ou avós. Estudos prévios sobre o tema, demonstram uma importância significativa da carga hereditária para o desenvolvimento da HAS. ${ }^{29,30}$

Destaca-se como principal ponto positivo deste estudo o seu tamanho amostral, incluindo trabalhadores de diferentes setores da indústria do estado do Rio Grande do Sul. O presente estudo adotou uma coleta padronizada dos dados, assim como todos os procedimentos necessários para a adequada aferição da pressão arterial. Ressalta-se, ainda, que procurou-se explorar os potenciais fatores associados à ocorrência de HAS, considerando a adoção de um modelo de análise multivariável. Contudo, os achados deste estudo devem ser interpretados considerando-se algumas limitações. Trata-se de um estudo com o uso de dados secundários, coletados entre os anos de 2006 e 2009, não incluindo a coleta de variáveis ocupacionais. Por se tratar de um estudo com desenho transversal, este pode estar sujeito a presença de causalidade reversa entre as associações investigadas. A presença do viés do trabalhador saudável ou sobrevivente também não pode ser totalmente descartada, uma vez que se consideraram no estudo apenas os trabalhadores ativos, ou seja, aqueles que estavam exercendo suas atividades laborais no momento de aplicação do estudo, excluindo 
Tabela 2 - Razões de prevalência (RP) brutas e ajustadas de hipertensão arterial sistêmica (HAS $\geq 140 / 90$ mmHg ou tratamento) segundo características demográficas, socioeconômicas, comportamentais, estado nutricional e hereditárias, na amostra de homens e mulheres trabalhadores da indústria do estado do Rio Grande do Sul, RS, Brasil, 2006-2009. (N=20.792)

\begin{tabular}{|c|c|c|c|c|c|}
\hline & & Hom & 349) & Mulhe & (8.443) \\
\hline & Características & RP Bruta* & RP Ajustada† & RP Bruta* & RP Ajustada† \\
\hline & Idade (anos) & $p<0,001$ & $p<0,001$ & $p<0,001$ & $p<0,001$ \\
\hline & 18 a 29 & 1 & 1 & 1 & 1 \\
\hline & 30 a 39 & $3,66(3,01-4,45)$ & $3,43(2,78-4,21)$ & $4,32(3,35-5,56)$ & $3,79(2,93-4,90)$ \\
\hline & 40 a 49 & $7,78(6,48-9,35)$ & $7,13(5,84-8,71)$ & $11,37(9,00-14,44)$ & $9,85(7,70-12,60)$ \\
\hline & 50 a 59 & $13,7(11,38-16,56)$ & $12,09(9,81-14,90)$ & $19,64(15,24-25,31)$ & $17,28(13,29-22,47)$ \\
\hline & Cor da pele & $\mathrm{p}=0,936$ & - & $\mathrm{p}=0,509$ & - \\
\hline & Branca & 1 & & 1 & \\
\hline & Não Branca & $1,01(0,89-1,13)$ & & $0,95(0,78-1,13)$ & \\
\hline & Situação conjugal & $p<0,001$ & $\mathrm{p}=0,028$ & $p<0,001$ & $p<0,001$ \\
\hline$\sum_{z}^{ \pm}$ & Sem companheiro & 1 & 1 & 1 & 1 \\
\hline$\frac{}{6}$ & Com companheiro & $2,27(1,98-2,60)$ & $1,17(1,02-1,35)$ & $1,50(1,28-1,76)$ & $1,28(1,09-1,51)$ \\
\hline 旁 & Escolaridade & $p<0,001$ & $p=0,182$ & $p<0,001$ & $p=0,001$ \\
\hline & Fundamental incompleto & $1,67(1,41-1,96)$ & $1,05(0,87-1,26)$ & $4,00(2,82-5,67)$ & $1,58(1,08-2,29)$ \\
\hline & Fundamental completo & $0,91(0,75-1,10)$ & $0,84(0,70-1,03)$ & $2,10(1,45-3,05)$ & $1,46(1,00-2,14)$ \\
\hline & Médio completo & $0,83(0,69-1,00)$ & $0,88(0,73-1,06)$ & $1,51(1,02-2,21)$ & $1,30(0,87-1,92)$ \\
\hline & Superior incompleto/completo & 1 & 1 & 1 & 1 \\
\hline & Renda familiar mensal (reais) & $p=0,005$ & $p=0,701$ & $p=0,002$ & $\mathrm{p}=0,055$ \\
\hline & $\leq 800$ & $0,83(0,72-0,95)$ & $0,96(0,83-1,12)$ & $1,50(1,19-1,88)$ & $1,30(1,02-1,66)$ \\
\hline & 801 a 1.200 & $0,77(0,67-0,89)$ & $0,89(0,77-1,03)$ & $1,35(1,07-1,69)$ & $1,20(0,95-1,51)$ \\
\hline & 1.201 a 1.800 & $0,78(0,67-0,90)$ & $0,89(0,76-1,03)$ & $1,40(1,09-1,79)$ & $1,16(0,91-1,49)$ \\
\hline & $>1.800$ & 1 & 1 & 1 & 1 \\
\hline & Tabagismo & $p<0,001$ & $p=0,262$ & $p=0,014$ & $p=0,133$ \\
\hline & Não fumante & 1 & 1 & 1 & 1 \\
\hline & Ex-fumante & $1,70(1,48-1,96)$ & $1,03(0,91-1,18)$ & $1,40(1,10-1,77)$ & $0,96(0,77-1,20)$ \\
\hline & Fumante & $1,23(1,08-1,40)$ & $1,08(0,95-1,23)$ & $0,91(0,71-1,16)$ & $0,83(0,67-1,05)$ \\
\hline & Consumo de álcool & $p=0,435$ & - & $p=0,002$ & $\mathrm{p}=0,824$ \\
\hline & Não consome & 1 & & 1 & 1 \\
\hline & Consome & $1,04(0,94-1,16)$ & & $0,67(0,52-0,86)$ & $0,98(0,77-1,25)$ \\
\hline & Atividade física & $p<0,001$ & $p=0,361$ & $\mathrm{p}=0,323$ & - \\
\hline$\sum_{z}^{\infty}$ & Ativo & 1 & 1 & 1 & \\
\hline 윰 & Inativo & $1,35(1,20-1,51)$ & $1,04(0,94-1,16)$ & $0,93(0,80-1,08)$ & \\
\hline 㤩 & Estado nutricional (IMC) & $p<0,001$ & $p<0,001$ & $p<0,001$ & $p<0,001$ \\
\hline & Normal $\left(<25 \mathrm{~kg} / \mathrm{m}^{2}\right)$ & 1 & 1 & 1 & 1 \\
\hline & Sobrepeso $\left(25\right.$ a $\left.29,9 \mathrm{~kg} / \mathrm{m}^{2}\right)$ & $2,78(2,42-3,18)$ & $1,94(1,69-2,22)$ & $3,34(2,78-4,02)$ & $2,04(1,69-2,45)$ \\
\hline & Obeso $\left(\geq 30 \mathrm{~kg} / \mathrm{m}^{2}\right)$ & $5,43(4,71-6,27)$ & $3,36(2,90-3,89)$ & $6,76(5,66-8,08)$ & $3,60(2,98-4,36)$ \\
\hline & Presença de HAS na família & $p<0,001$ & $p<0,001$ & $p<0,001$ & $p<0,001$ \\
\hline & Não & 1 & 1 & 1 & 1 \\
\hline & Mãe ou Pai & $1,61(1,44-1,80)$ & $1,49(1,34-1,65)$ & $2,17(1,82-2,58)$ & $1,78(1,50-2,10)$ \\
\hline & Mãe e Pai & $2,94(2,50-3,46)$ & $2,16(1,86-2,52)$ & $4,68(3,81-5,76)$ & $2,77(2,27-3,38)$ \\
\hline & Mãe, Pai e Avós & $3,31(2,61-4,19)$ & $2,95(2,40-3,62)$ & $4,47(3,48-5,74)$ & $3,22(2,54-4,07)$ \\
\hline
\end{tabular}

* p-valor para teste Wald para heterogeneidade de proporções (variáveis categóricas) ou tendência linear (variáveis ordinais) obtido por meio da regressão de Poisson com variância robusta; † Análise ajustada por modelo multivariável, incluindo as variáveis com p<0,20 na análise bruta. Primeiro Nivel: ajuste entre as variáveis sociodemográficas; Segundo Nivel: ajuste entre as variáveis do primeiro nivel com p<0,20 mais as variáveis comportamentais, estado nutricional e hereditariedade. 
os que se encontravam afastados por algum fator de saúde, por exemplo. Outra limitação do estudo deve-se à falta de uma análise de variabilidade intra e interobservador para as mensurações das pressões sistólicas e diastólicas obtidas. Por fim, a realização de análises por setores da indústria, assim como possíveis diferenças sociodemográficas dentre os setores, ficaram prejudicadas em decorrência da grande heterogeneidade do número de trabalhadores investigados em cada setor.

Embora a prevalência de HAS encontrada em nosso estudo tenha sido relativamente baixa, observou-se que a ocorrência de HAS em trabalhadores da indústria é influenciada por fatores sociodemográficos, nutricionais e de história familiar. Contudo, é pertinente que outros estudos sejam realizados, tendo como objetivo identificar os potenciais fatores de risco ocupacionais (tipo de ocupação; turno de trabalho; tempo de serviço; presença de programa alimentar no trabalho; entre outros) associados a HAS, visando a medidas e ações específicas voltadas para a ocorrência de doenças crônicas não transmissíveis, e focadas na saúde integral do trabalhador.

\section{Conclusões}

Nossos achados, na amostra estudada, apontaram que fatores sociodemográficos, nutricionais e de história familiar estão associados à ocorrência de HAS. Identificou-se que a prevalência de HAS aumentou conforme o avanço da idade e situação conjugal, em ambos os sexos. De maneira semelhante, as condições socioeconômicas desfavoráveis

\section{Referências}

1. Coelho EF, Ferreira RE, Oliveira TFB, Navarro CA, Ferreira RM, Vianna JM, et al. Prevalência de fatores de risco para doença cardiovascular em trabalhadores de empresa siderúrgica. Rev Bras Ciên Saúde. 2014;18(Supl 4):51-8. DOI: 10.4034/rbcs.2014.18.S4.07

2. Psaltopoulou T, Hatzis G, Papageorgiou N, Androulakis E, Briasoulis A, Tousoulis D. Socioeconomic status and risk factors for cardiovascular disease: Impact of dietary mediators. Hellenic J Cardiol. 2017;58(1):32-42. DOI: 10.1016/j.hjc.2017.01.022.

3. Montalti M, Zanobini A, Luchetti E, Arcangeli G. 827 Arterial hypertension risk in a working population.7Occupational and Environmental Med. 2018; 75(Suppl 2):A 65-A2-65. DOI: 10.1136/oemed-2018-icohabstracts.186.

4. Malachias MV. 7th brazilian guideline of arterial hypertension: Presentation Arq Bras Cardiol. 2016;107(3 Suppl 3). DOI: 10.5935/abc.20160140.

5. World Health Organization.(WHO) Global Status Report on Noncommunicable diseases. Geneva ;2014

6. Organização Pan Americana da Saúde. (OPAS)Análisis de salud, métricas y evidencia: Situación de salud en las américas: Indicadores básicos 2018. Washington, D.C., Estados Unidos de América; 2018. Available from: http:// www.paho.org/data/index.php/es/\%0A223435_ESPANOL.indd.1-20.

7. Vinholes DB, Bassanesi SL, Chaves Junior HC, Machado CA, Melo IMF, Fuchs FD, et al. Association of workplace and population characteristics with prevalence of hypertension among Brazilian industry workers: a multilevel analysis. BMJ Open. 2017;7(8):e015755. DOI: 10.1136/ bmjopen-2016-015755.

8. Cassani RS, Nobre F, Pazin Filho A, Schmidt A. Prevalence of cardiovascular risk factors in a population of Brazilian industry workers. Arq Bras Cardiol. 2009;92(1):16-22. DOI: 10.1590/s0066-782×2009000100004. se associaram à presença de HAS, principalmente entre as mulheres. Tal evidência pode contribuir na formulação de intervenções efetivas para a prevenção da HAS junto às políticas voltadas à saúde do trabalhador.

\section{Contribuição dos autores}

Concepção e desenho da pesquisa: Xavier PB, Cibeira GH, Germano A, Olinto MTA; Obtenção de dados: Xavier PB, Cibeira GH, Germano A; Análise e interpretação dos dados e Análise estatística: Xavier PB, Garcez A, Olinto MTA; Redação do manuscrito e Revisão crítica do manuscrito quanto ao conteúdo intelectual importante: Xavier PB, Garcez A, Cibeira GH, Germano A, Olinto MTA.

\section{Potencial conflito de interesse}

Não há conflito com o presente artigo

\section{Fontes de financiamento}

O presente estudo não teve fontes de financiamento externas.

\section{Vinculação acadêmica}

Este artigo é parte de tese de doutorado de Paula Brustolin Xavier pela Universidade do Vale do Rio dos Sinos (UNISINOS).

9. Wang L, Rosenman K. Adverse Health Outcomes Among Industrial and Occupational Sectors in Michigan. Prev Chronic Dis. 2018;15(8):E102. DOI: $10.5888 /$ pcd15.170487.

10. Doubova SV, Sanchez-Garcia S, Infante-Castaneda C, Perez-Cuevas R. Factors associated with regular physical exercise and consumption of fruits and vegetables among Mexican older adults. BMC Public Health. 2016;16(1):952. DOI: 10.1186/s12889-016-3628-2.

11. Limaye TY, Kulkarni RL, Deokar MR, Kumaran K. High prevalence of cardiometabolic risk factors in young employees of information technology industry. Indian J Occup Environ Med. 2016;20(1):64-7. DOI: 10.4103/0019-5278.183848.

12. Dantas J, Mendes R, Araújo TMd. Hipertensão arterial e fatores psicossociais no trabalho em uma refinaria de petróleo. Rev Bras Med Trab. $2004 \cdot 2(1): 55-68$

13. Martinez MC, Latorre MO. Fatores de risco para hipertensão arterial e diabete melito em trabalhadores de empresa metalúrgica e siderúrgica. Arq Bras Cardiol. 2006;87(4):471-9. DOI: 10.1590/S0066-782X2006001700012.

14. World Health Organizarion.(WHO). Physical status: the use and interpretation of anthropometry.Geneva;1995.[Cited in 2020 Jan 12 ] Available from: https://apps.who.int/iris/bitstream/handle/10665/37003/ WHO_TRS_854.pdf;jsessionid=304EB92FBC2312243C1628A1C1E02 875 ? sequence $=1$.

15. Kohlmann Jr O, Costa Guimarães A, Carvalho MHC, Chaves Jr HC, Machado CA, Praxedes JN, et al. III Consenso Brasileiro de Hipertensão Arterial. Arq Bras Endocrinol Metab. 1999;43(4):257-86. DOI: 10.1590/S000427301999000400004. 
16. Salem H, Hasan DM, Eameash A, El-Mageed HA, Hasan S, Ali R. Worldwide prevalence of hypertension: A pooled meta-analysis of 1670 studies in 71 countries with 29.5 million participants. J Am Coll Cardiol. 2018;71(11):A1819 DOI: 10.1016/s0735-1097(18)32360-x.

17. Barros AJ, Hirakata VN. Alternatives for logistic regression in crosssectional studies: an empirical comparison of models that directly estimate the prevalence ratio. BMC Med Res Methodol. 2003;3(1):21. DOI: 10.1186/1471-2288-3-21.

18. Victora CG, Huttly SR, Fuchs SC, Olinto MT. The role of conceptual frameworks in epidemiological analysis: a hierarchical approach. Int J Epidemiol. 1997;26(1):224-7. DOI: 10.1093/ije/26.1.224.

19. Zhou B, Bentham J, Di Cesare M, Bixby H, Danaei G, Cowan MJ, et al. Worldwide trends in blood pressure from 1975 to 2015: a pooled analysis of 1479 population-based measurement studies with $19 \cdot 1$ million participants. Lancet. 2017;389(10064):37-55. DOI: 10.1016/s0140-6736(16)31919-5.

20. Paquissi FC, Manuel V, Manuel A, Mateus GL, David B, Beu G, et al. Prevalence of cardiovascular risk factors among workers at a private tertiary center in Angola. Vasc Health Risk Manag. 2016;12:497-503. DOI: 10.2147/VHRM.S120735.

21. Barbini N, Speziale M, Squadroni R. Occupational risk factors for arterial hypertension in workers of high speed railway line in Italy. Arch Clin Hypert. 2017;3(1):001-004. DOI: 10.17352/ach.000011.

22. Silva SS, Oliveira Sde F, Pierin AM. O controle da hipertensao arterial em mulheres e homens: uma analise comparative. Rev Esc Enferm USP. 2016;50(1):50-8. DOI: 10.1590/S0080-623420160000100007.
23. Clougherty JE, Souza K, Cullen MR. Work and its role in shaping the social gradient in health. Ann N Y Acad Sci. 2010;1186(1):102-24. DOI: 10.1111/j.1749-6632.2009.05338.x.

24. Lobo LAC, Canuto R, Dias-da-Costa JS, Pattussi MP. Tendência temporal da prevalência de hipertensão arterial sistêmica no Brasil. Cad Saúde Pública. 2017;33(6):e00035316. DOI: 10.1590/0102-311X00035316.

25. Krieger N. Genders, sexes, and health: what are the connections--and why does it matter? Int J Epidemiol. 2003;32(4):652-7. DOI: 10.1093/ije/dyg156.

26. Mattei TF, Baço FMB. Análise da existência de discriminação salarial entre homens e mulheres na indústria de transformação do estado de santa catarina. Rev E\&G. 2016;16(45):103-25. DOI: 10.5752/P.19846606.2016v16n45p103.

27. Obarisiagbon OE, Osayi D, Wagbatsoma VA. Prevalence and risk factors of hypertension among workers of an oil palm company in Edo State, Nigeria. J Comm Med Prim Health Care. 2018;30(2):62-74.

28. Linhares RS, Horta BL, Gigante DP, Dias-da-Costa JS, Olinto MT. Distribuição de obesidade geral e abdominal em adultos de uma cidade no Sul do Brasil. Cad Saúde Pública. 2012;28(3):438-47. DOI: 10.1590/s0102$311 \times 2012000300004$

29. de Oliveira CM, Pereira AC, de Andrade M, Soler JM, Krieger JE. Heritability of cardiovascular risk factors in a brazilian population: Baependi heart study. BMC Med Genet. 2008;9:32. DOI: 10.1186/1471-2350-9-32.

30. Ranasinghe P, Cooray DN, Jayawardena R, Katulanda P. The influence of family history of hypertension on disease prevalence and associated metabolic risk factors among Sri Lankan adults. BMC Public Health. 2015;15:576. DOI: 10.1186/s12889-015-1927-7. 